\title{
Effect of Dynamic Sector Boundary Changes on Air Traffic Controllers
}

\author{
Jaewoo Jung* \\ University of California Santa Cruz, NASA Ames Research Center, Moffett Field, CA 94035 \\ Paul Lee, ${ }^{\dagger}$ Angela Kessell ${ }^{\dagger}$ and Jeff Homola ${ }^{\S}$ \\ San Jose State University, NASA Ames Research Center, Moffett Field, CA 94035 \\ and \\ Shannon Zelinski ${ }^{\top}$ \\ NASA Ames Research Center, Moffett Field, CA 94035
}

The effect of dynamic sector boundary changes on air traffic controller workload was investigated with data from a human-in-the-loop simulation. Multiple boundary changes were made during simulated operations, and controller rating of workload was recorded. Analysis of these data showed an increase of $16.9 \%$ in controller workload due to boundary changes. This increased workload was correlated with the number of aircraft handoffs and change in sector volume. There was also a $12.7 \%$ increase in average workload due to the changed sector design after boundary changes. This increase was correlated to traffic flow crossing points getting closer to sector boundaries and an increase in the number of flights with short dwell time in a sector. This study has identified some of the factors that affect controller workload when sector boundaries are changed, but more research is needed to better understand their relationships.

$\begin{array}{ll}\text { Nomenclature } \\ A T F_{b c} & =\text { airspace transition workload multiplier during a boundary change } \\ A T F_{s s} & =\text { airspace transition workload multiplier after a boundary change (steady state) } \\ R & =\text { correlation coefficient } \\ W_{N b c} & =\text { average of nominal workloads during a boundary change } \\ W_{N s s} & =\text { average of nominal workloads after a boundary change (steady state) } \\ W_{R b c} & =\text { average of recorded controller workloads during a boundary change } \\ W_{R s s} & =\text { average of recorded controller workloads after a boundary change (steady state) }\end{array}$

\section{Introduction}

$\mathrm{T}$ HE airspace of the contiguous United States is divided into 20 air route traffic control centers. Each center is divided further into sectors, where the shapes and sizes of sectors are configured to equitably distribute air traffic control workload during nominal conditions. ${ }^{1,2}$ To maintain the equitable distribution of workload, sector boundaries can be dynamically changed during off-nominal conditions, such as when flights from weather-affected sectors are routed through adjacent sectors. ${ }^{3-8}$

For dynamic sector boundary changes to be used in operation, it is necessary to understand their impact on air traffic controller workload. Compared to a fixed sector boundary operation, dynamically changing boundaries adds more responsibilities to the controllers. The additional responsibilities include handing off aircraft to the controller

\footnotetext{
* Associate Scientist, Systems Modeling and Optimization Branch, NASA ARC, MS 210-8, Senior Member.

${ }^{\dagger}$ Senior Research Associate, Airspace Operations Laboratory, NASA ARC, MS 262-4.

* Research Associate, Airspace Operations Laboratory, NASA ARC, MS 262-4.

${ }^{\S}$ Research Associate, Airspace Operations Laboratory, NASA ARC, MS 262-4.

"Aerospace Engineer, Systems Modeling and Optimization Branch, NASA ARC, MS 210-10, Member.
} 
of the new sector that the aircraft would be in after a boundary change and maintaining situational awareness through the changes. Studies from a human factors perspective indicated that dynamic boundary changes might have a negative impact on controller situational awareness and performance when implemented without careful preparation or controller training. ${ }^{9}, 10$ Pre-defined boundary configurations, pre-determined times to change, and controller training for the configurations were used in these studies.

To obtain further insight into the effect of dynamic boundary changes on air traffic controller workload, a human-in-the-loop simulation was conducted at NASA in May 2009. Multiple boundary changes were made during simulated traffic control operations, and controller's perception of workload rating was recorded. The controllers had no previous knowledge of scheduled boundary changes. They were told of a boundary change three-minutes prior to the change. This paper examines the impact of such boundary changes on air traffic controller workload by analyzing data from the simulation.

The paper is organized as follows. Section II contains a brief description of the human-in-the-loop simulation. Section III presents the analysis of data collected from the simulation. In Section IV the paper is concluded and recommendations are made for future research.

\section{Brief Description of the Human-in-the-Loop Simulation}

In May 2009, a human-in-the-loop (HITL) simulation was conducted at NASA to obtain insight into the effect of dynamic boundary changes on air traffic controller workload. The following subsections describe the scenarios, setup, and recorded data for this simulation.

\section{A. Simulation Scenarios}

In the HITL simulation, four adjacent high altitude sectors in the Kansas City air route traffic control center, ZKC 29, 90, 94 and 98, were subjected to boundary changes. Four 60-minute scenarios were used in the simulation. For all scenarios, the average aircraft count in sectors ZKC 90 and 94 were set to be higher than the ones in ZKC 29 and 98. Such difference was used to create an unequal distribution of workload among the sectors. Dynamic sector boundary changes were used to re-distribute the workload equitably among the sectors. Simulation runs with the same scenarios without the boundary changes were used as the baseline.

Three algorithms, based on dynamic airspace units, ${ }^{11}$ flight clustering, ${ }^{12}$ and mixed integer programming, ${ }^{13}$ were used to generate new boundaries. Inputs for the algorithms were the traffic data and the original sector boundary configurations. The objective for all algorithms was to distribute aircraft counts equitably among the sectors, therefore emulating equitable distribution of workload. Figure 1 shows a result of boundary changes. The difference in the average aircraft count between ZKC 90 and 98 in the baseline (top plot) became less with boundary changes (bottom plot).
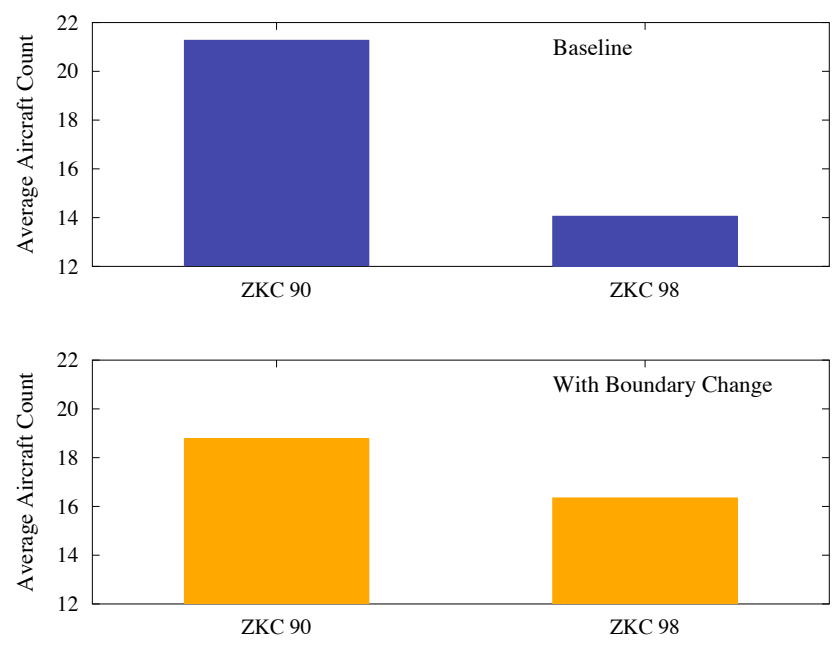

Figure 1. The average aircraft count in sectors ZKC 90 and 98 , baseline and with boundary changes.

\section{B. Simulation Setup and Recorded Data}

An area supervisor and six air traffic controllers participated in the HITL simulation. A single controller was assigned to each of the four sectors. The area supervisor decided when and which sector required aid from one or two additional controllers. In a typical run, three boundary changes occurred. Boundary configurations, except ones at the start time, were unknown to the controllers. A preview of the upcoming boundary change was displayed to the controllers three minutes prior to the change. Only boundaries between sectors were changed. Figure 2 shows examples of boundary changes. 
A self-rating of each controller's perception of workload was recorded in five minute intervals and at three and one minutes before and after each boundary change. The same times were used to record workload for the baseline. For simplicity, self-rated controller's perception of workload is referred to as controller workload from now on.

The workload rating ranged from one to seven on an integer scale, where seven indicated the highest workload. At each recording time, controllers were asked to assess their workload level and press a button on a numeric keyboard. Each recording time was announced by an audio cue. Workload recordings were not collected at the exact time of boundary changes so that the controllers would not be distracted. Late transfers were allowed, i.e. an aircraft was allowed to cross a sector boundary first, and have its radar identification transferred afterwards.

The simulation produced data for 36 boundary change cases (4 scenarios x 3 boundary changes per scenario $\mathrm{x} 3$ boundary change algorithms), and for the corresponding baseline cases. For a complete description of the HITL simulation, readers are referred to Ref. 14. Details of the simulation setup, such as specific boundary change times of each scenario, are available in the reference.

\section{Analysis of the Simulation Data}

In this section, the analysis of simulation data is presented in two subsections. The first subsection examines the effect of dynamic sector boundary changes on air traffic controller workload during the boundary changes. The second subsection examines the effect of the changes on the average workload after the changes.

\section{A. Effect of Boundary Changes on Controller Workload During the Boundary Changes}

In the HITL simulation, the controllers were responsible for handing off aircraft to the controller of the new sector that the aircraft would be in after a boundary change. The controllers were instructed to arrange and complete the handoffs within the three-minute boundary change preview period. Also, the controllers were responsible for maintaining situational awareness and providing safe separation of the traffic through the boundary changes. Compared to the baseline, both responsibilities were additional burdens on the controllers. Therefore, it was hypothesized that dynamic sector boundary changes would increase the workload of controllers during the changes.

To test the hypothesis, workload recordings during the boundary changes were compared to the ones from the baseline. Even though there were no boundary changes in the baseline, workloads were recorded at the same interval as the boundary change cases. The average of workload recordings at three and one minutes before and after a boundary change was used to capture workload during the change. Figure 3 shows distribution of workload during boundary changes, where occurrence of a particular workload is shown as frequency in the y-axis. The figure shows a clear increase in the controller workload during the sector boundary changes.
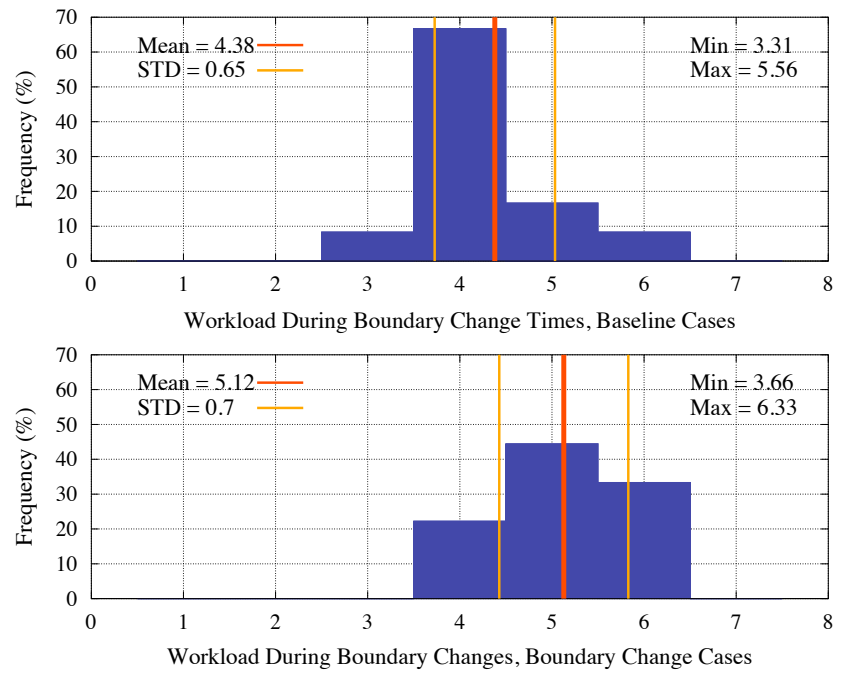

Figure 3. Distribution of workload during boundary changes.

American Institute of Aeronautics and Astronautics 
Compared to the baseline, the mean workload during the changes was $16.9 \%$ higher with the boundary changes, and the distribution of the workload showed a bias toward the higher ratings. The comparison agreed with the hypothesis.

This result showed that dynamic sector boundary changes increased controller workload during the changes. One can model such increase in workload with the following equation:

$$
A T F_{b c}=\frac{W_{R b c}}{W_{N b c}}
$$

where $W_{R b c}, W_{N b c}$, and $A T F_{b c}$ are the average of recorded controller workloads at three and one minutes before and after a boundary change, the average of baseline workloads at the same times, and airspace transition workload multiplier during the boundary change, respectively.

To understand reasons for the increase in workload during the boundary changes, relationships between $A T F_{b c}$ and several airspace transition metrics were examined through a regression analysis. Results of the analysis showed that $A T F_{b c}$ is related to a change in sector volume. Figure 4 shows the relation with a linear fit. The results also showed that sector volume change is correlated with the number of aircraft handoffs, with $\mathrm{R}^{2}$ of 0.74 . These results indicated that when a boundary change caused sector volumes to change, number of aircraft handoffs increased in proportion to the volume change. Controller workload during the change increased in proportion to both metrics.

The analysis results matched with an observation made during the simulation, that the more drastic the changes in the sector boundaries, the more difficulties the controllers had. For example, the number of controller remarks indicating frustration was highly related to visually observed magnitude of the boundary changes (i.e. related to sector volume change).

The analysis results also matched with the current practice of sector configuration change, where the change is implemented when there is a gap in the traffic flow, thereby reducing number of aircraft handoffs. For future studies, additional insight on causes of workload increase during the changes can be gained by de-coupling change in sector volume from number of aircraft handoffs. Implementing boundary changes when there are no aircraft near sector boundaries might be worth investigating.

\section{B. Effect of Boundary Changes on the Average Controller Workload after the Changes}

The dynamic boundary changes affected the relation between air traffic routes and the sectors that contained them. It was assumed that the original sectors were designed to contain the routes in a manner that was favored by the controllers. With this assumption, it was hypothesized that the boundary changes would increase average controller workload after the changes, as the boundary changes altered alignment between the traffic and the airspace.

To test the hypothesis, workload recordings from the boundary change cases were compared with the ones from the baseline. The average of the workload recordings from 5 minutes after a boundary change and 5 minutes before the next change was used as steady state workload. For simplicity, average controller workload between boundary changes is referred to as steady state workload from now on. Figure 5 shows an increase in steady state workload. Compared to the baseline, the mean steady state workload was $12.7 \%$ higher with the boundary changes. The comparison agreed with the hypothesis.

This result showed that the boundary change increased steady state workload. To model the increased workload, the following equation is used:

$$
A T F_{s s}=\frac{W_{R s s}}{W_{N s s}}
$$


where $W_{R s s}, W_{N s s}$, and $A T F_{s s}$ are recorded steady state controller workload with boundary changes, the baseline steady state workload, and steady state airspace transition workload multiplier, respectively.

To understand reasons for the increase in steady state workload, relationships between $A T F_{s s}$ and several airspace design metrics were examined through a regression analysis. Differences between the metrics from the boundary change cases and the corresponding baseline cases were used as independent variables for the regression analysis.

Results of the analysis indicated that $A T F_{s s}$, and therefore the steady state workload, increased when the average distance between sector boundaries and traffic flow crossing points became smaller and when the number of flights with short dwell time in a sector became larger. Short dwell time flights were ones with dwell time of less than a minute. Figure 6 shows the relation and $\mathrm{R}^{2}$ of each metric to $A T F_{s s}$. Correlation between the metrics was insignificant, with $\mathrm{R}^{2}$ of 0.04 .

The analysis results showed that boundary changes can be detrimental to sector design, causing an increase in the average workload after the changes. Therefore, sector design factors, especially ones that determine the relation between air traffic routes and the sectors that contain them, should be considered when sector boundary changes are planned.

Times between the boundary changes in the simulation were 15 to 30 minutes. For future studies, increased time between the boundary changes is recommended to gain further insight into the effect of boundary changes on steady state workload.

\section{Conclusion}

An analysis was performed on data from a human-in-the-loop simulation to obtain insight into the effect of dynamic sector boundary changes on air traffic controller workload. In the simulation, multiple boundary changes were made during operation, and controller workload ratings were recorded. The simulation used traffic scenarios where workloads of certain sectors were set to be much higher than their adjacent sectors. Boundary change algorithms were used to distribute the workload.

Whereas the boundary change succeeded in distributing the sector aircraft count equitably, increase in workload was found when boundary change cases were compared to baseline cases with the same traffic conditions without boundary changes. Workload during the changes was $16.9 \%$ higher, and average workload after the changes was $12.7 \%$ higher than the baseline cases.

Further analysis showed that increased workload during boundary changes was correlated to increased sector volume change. Increased average workload after the changes was correlated to two factors. The workload increased when traffic flow crossing points got closer to sector boundaries and when the number of flights in a sector with less than a minute of dwell time became larger. These correlations suggest that these sector design factors should be considered when boundary changes are planned. 
Due to the simulation setup, sector volume change was related with the number of aircraft handoffs at each boundary change. De-coupling the two factors is recommended for future studies by implementing boundary changes when there are no aircraft near sector boundaries. Also, increasing time between the boundary changes is recommended to gain further insight into the effect of boundary changes on steady state workload.

\section{Acknowledgments}

The authors would like to thank Michael Bloem for helping with developing airspace transition and design metrics, Hwasoo Lee for providing simulation data, Michael Jastrzebski and Chok Fung Lai for metrics calculation, and Michael Drew for feedback on the data analysis.

\section{References} 1976.

${ }^{1}$ Schmidt, David K., “On Modeling ATC Work Load and Sector Capacity,” Journal of Aircraft, Vol. 13, No. 7, 531-537, July

${ }^{2}$ Federal Aviation Administration, Air Traffic Organization Policy, SUBJ: facility Operation and Administration, U.S. Department of Transportation, ORDER JO 7210.3W, p. 6-1-1, 2010.

${ }^{3}$ Kopardekar, Parimal, Karl Bilimoria and Banavar Sridhar, "Initial Concepts for Dynamic Airspace Configuration," AIAA 2007-7763, $7^{\text {th }}$ AIAA Aviation Technology, Integration and Operations Conference, Belfast, Northern Ireland, Sep. 2007.

${ }^{4}$ Klein, Alexander, Parimal Kopardekar, Mark D Rogers and Hong Kaing, “'Airspace Playbook': Dynamic Airspace Reallocation Coordinated with the National Severe Weather Playbook," AIAA 2007-7764, $7^{\text {th }}$ AIAA Aviation Technology, Integration and Operations Conference, Belfast, Northern Ireland, Sep. 2007.

${ }^{5}$ Brinton, Chris R and Lara S. Cook, "Analysis of Current Airspace Operation and Implications for Dynamic Airspace Configuration," AIAA 2008-7224, AIAA Guidance, Navigation, and Control Conference and Exhibit, Honolulu, Hawaii, Aug. 2008.

${ }^{6}$ Lee, Paul U., Joey Mercer, Brian F. Gore, Nancy Smith, Katharine Lee and Robert Hoffman, "Examining Airspace Structural Components and Configuration Practices for Dynamic Airspace Configuration," AIAA 2008-7228, AIAA Guidance, Navigation, and Control Conference and Exhibit, Honolulu, Hawaii, Aug. 2008.

${ }^{7}$ Doble, Nathan A., Robert Hoffman, Paul U. Lee, Joey Mercer, Brian Gore, Nancy Smith and Katharine Lee, "Current Airspace Configuration Practices and Their Implications for Future Airspace Concepts," AIAA 2008-8936, 26 ${ }^{\text {th }}$ Congress of International Council of the Aeronautical Sciences, Anchorage, Alaska, Sep. 2008.

"Limited Dynamic Resectorization Casebook," MITRE Corporation Center for Advanced Aviation System Development, McLean, VA, 2000.

${ }^{9}$ Stein, Earl S., Pamela S. Della Rocco and Randy L. Sollenberger, "Dynamic Resectorization in Air Traffic control: A Human Factors Perspective," U.S. Department of Transportation/Federal Aviation Administration Report Number DOT/FAA/TC-TN06/19, May 2006.

${ }^{10}$ Hadley, Jerry, Randy Sollenberger, Jean-Francois D'Arcy and Philip Bassett, "Interfacility Boundary Adjustment," U.S. Department of Transportation/Federal Aviation Administration Report Number DOT/FAA/CT-TN00/06, July 2000.

${ }^{11}$ Klein, Alexander., Mark Rogers and Hong Kaing, "Dynamic FPAs: A New Method for Dynamic Airspace Configuration," Integrated Communications Navigation and Surveillance (ICNS) Conference. Bethesda, MD, 2008.

${ }^{12}$ Brinton, Chris R. and Stephen Pledgie, “Airspace Partitioning using Flight Clustering and Computational Geometry,” In Proceedings of the 27th Digital Avionics Systems Conference (DASC), St. Paul, MN, 2008.

${ }^{13}$ Yousefi, Arash, "Optimum Airspace Design with Air Traffic Controller Workload-based Partitioning," Ph.D. dissertation, George Mason University, 2005.

${ }^{14}$ Homola, Jeffery, Paul U. Lee, Thomas Prevot, Hwasoo Lee, Angela Kessell, Connie Brasil and Nancy Smith, "A Humanin-the Loop Exploration of the Dynamic Airspace Configuration Concept," AIAA GNC 2010, Toronto, Ontario, Canada, AIAA (submitted for publication) 